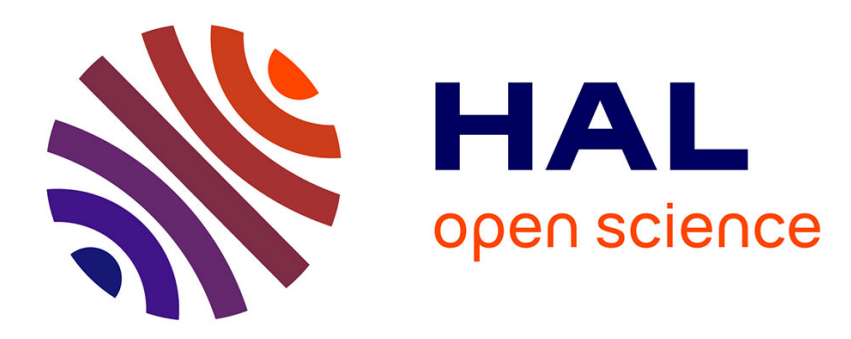

\title{
La négociation contre la démocratie : le cas Wikipedia \\ Pierre-Carl Langlais
}

\section{To cite this version:}

Pierre-Carl Langlais. La négociation contre la démocratie : le cas Wikipedia. Négociations, 2014, Démocratie et négociations, 21, pp.21-34. 10.3917/neg.021.0021 . sic_01222973

\section{HAL Id: sic_01222973 \\ https://archivesic.ccsd.cnrs.fr/sic_01222973}

Submitted on 31 Oct 2015

HAL is a multi-disciplinary open access archive for the deposit and dissemination of scientific research documents, whether they are published or not. The documents may come from teaching and research institutions in France or abroad, or from public or private research centers

\section{(1) (0)}

Distributed under a Creative Commons CCO - Public Domain Dedication| 4.0 International
L'archive ouverte pluridisciplinaire HAL, est destinée au dépôt et à la diffusion de documents scientifiques de niveau recherche, publiés ou non, émanant des établissements d'enseignement et de recherche français ou étrangers, des laboratoires publics ou privés. 
La négociation contre la démocratie : le cas Wikipedia

Pierre-Carl Langlais ${ }^{1}$ GRIPIC / Paris-Sorbonne

Mots-clés : Communautés en ligne - Consensus - Démocratie consensuelle Démocratie majoritaire - Arend Lijphart — Wikipédia

Le premier principe fondateur de Wikipédia souligne que «Wikipédia n'est pas une démocratie ». Les communautés wikipédiennes tendent à ériger la négociation en contremodèle de la démocratie et de la voie des urnes. Cet article s'interroge sur la validité et les motifs de cette conception spécifique. S'appuyant sur le cadre d'analyse élaboré par Arend Lijphart, il met en évidence l'émergence d'un modèle de gouvernance mixte, intégrant des éléments de démocratie majoritaire dans le cadre d'une démocratie consensuelle. Le rejet de l'interprétation démocratique a une utilité sociale : il permet d'appréhender les procédures politiques traditionnelles sur un mode exclusivement pragmatique.

Keywords : Computer mediated community - Consensus - Consensual Democracy — Majoritarian Democracy — Arend Lijphart — Wikipedia

The first pillar of Wikipedia stresses that "Wikipedia is not a democracy ». The wikipedian communities tend to view democracy and polling as the alter ego (if not the nemesis) of negociation and consensual thought. This article questions the validity and the motives of such a specific conception. Using the conceptual framework of Arend Lijphart, it describes the emergence of a joint-system, which includes elements of majoritarian democracy into the general setting of a consensual democracy. The unconditional rejection of democratic interpretation seems to have its own social use : it allows a pragmatic acclimation of pre-existent procedures in the static political system.

${ }^{1}$ pierre-carl.langlais@orange.fr 
«Wikipédia n'est pas une démocratie » / «Wikipédia n’est pas une expérience politique $»^{2}$. Telles sont les injonctions que les communautés wikipédiennes développent dans leur premier principe fondateur, sous le titre générique : "Wikipédia est une encyclopédie ॥. Le message a le mérite d'être clair : Wikipédia se présente d'emblée comme une entité apolitique.

Quelques recommandations plus détaillées étayent cette affirmation péremptoire. La page anglophone Ce que Wikipédia n'est pas (What Wikipedia is not) comporte ainsi la précision suivante :

Wikipédia n'est pas une expérience démocratique ni ne prétend s'apparenter à aucun autre système politique. La prise de décision et la résolution des conflits passe par deux processus fondamentaux (quoique non exclusifs) : l'édition des articles et l'exercice d'une discussion consensuelle - et non pas le vote. ${ }^{3}$

Ces deux phrases nécessitent une lecture attentive. Le rejet du politique sous toutes ces formes est confirmé. "Aucun autre système politique » : il ne s'agit pas seulement de contester toute analogie avec le système démocratique mais bel et bien la pertinence de l'interprétation politique dans l'absolu.

S'ensuit une brève description du mode de régulation alternatif promu par la communauté encyclopédique. Deux attributs fondamentaux de l'autorité politique (la prise de décision et la résolution des conflits) sont dévolus à des procédés techniques (l'édition des articles) ou à des pratiques sociales (la délibération et le recours au consensus informel entre participants rationnels). Quant au vote, sur lequel repose une bonne part de la légitimité des États démocratiques, il est rejeté avec force : « et non pas le vote ».

En creux, on voit se dessiner une conception restreinte du politique. Le vote majoritaire fonderait une épistémologie spécifiquement politique, faisant primer la conception la plus répandue dans une population donnée. Wikipédia proposerait, par contraste, une

\footnotetext{
2 «Wikipedia:Five Pillars », en.wikipedia.org, version du 15 décembre 2012 (http://pprr.it/e2l)

${ }^{3}$ Extrait de la page «What Wikipedia is not » de en.wikipedia.org, version du 13 janvier 2012 (http://ppfr.it/ e2m)
} 
épistémologie concurrente, reposant non sur le constat d'une opinion publique mais sur l'exercice continu d'une rationalité délibérative. Les règles communautaires opposent ainsi une culture communicationnelle du consensus à une culture agonistique de la sphère politique, une société de connaissances à une société de pouvoirs. Dans cette constellation terminologique particulière, la négociation devient l'autre de la démocratie : elle tient à la fois lieu d'antithèse et d'alternative.

\section{Wikipédia comme démocratie délibérative?}

Les recherches dédiées à la gouvernance wikipédienne sont rares. Sur les centaines de publications académiques référencées par le site WikiPapers, guère plus d'une dizaine relèvent expressément de cette thématique ${ }^{4}$. Aucune d'entre elle n'est conçue par un politologue : on note une prédominance des recherches en sciences de l'information (Reagle, 2010, Geiger, 2007, Butler et al., 2008, Black et al., 2008) et en sociologie (Cardon et Levrel, 2009, Auray et al., 2009).

Ce désintérêt témoigne de la réticence des chercheurs en science politique à s'approprier des objets trop éloignés de leurs préoccupations courantes. La plupart des études politiques consacrées à Internet partagent un biais commun : elles s'interrogent exclusivement sur les effets des réseaux numériques sur le système politique formel et ses acteurs institutionnalisés (Vedel, 2006 : 53). En se focalisant sur l'acclimatation numérique des acteurs traditionnels, elles laissent de côté les phénomènes de gouvernementalité intrinsèque des communautés en ligne (Black et al., 2008 : 1).

On en vient ainsi à constater un curieux décalage : si en théorie Wikipédia peut être considérée comme une expérience politique au même titre que toute société humaine d'une certaine amplitude, cette dimension de l'encyclopédie reste peu étudiée. S'y intéresser suppose en effet de renouveler plusieurs cadres usuels de compréhension. L'étude de Laura Black et al. (2008 : 1) développe une piste intéressante en se référant à la démocratie délibérative de Robert Dahl. Elle amorce un rapprochement avec un champ de recherche très actif depuis quelques décennies : les études sur la négociation.

Par-delà leur diversité, ces études préconisent un élargissement des conceptions usuelles de la démocratie et de la vie politique. Elles se situent au carrefour de plusieurs sciences sociales (économie, politique, diplomatique, sociologie), qu'elles font couramment dialoguer avec des approches formalistes (notamment en provenance de la

\footnotetext{
${ }^{4} \mathrm{Cf}$. notamment la page « List of research areas » du site wikipapers.referata.com, version du 8 novembre 2012 (http://ppfr.it/e2n)
} 
théorie des jeux). Depuis le grand tournant empirique des années 1960, l'accent est mis sur l'analyse de situations concrètes, où la négociation se trouve structurée d'avance par des règles préexistantes, plus ou moins formalisées (Touzard, 2009 : 60-61).

Dans ce courant, la négociation n'est pas qu'un concept théorique. Elle renvoie à une dynamique historique caractéristique des sociétés contemporaines, comme en témoigne la formule, célèbre, de Zartman (1976 : 2-3) est sans équivoque : « Nous vivons à l'âge de la négociation (...) La négociation ne devient pas seulement un processus mais un mode de vie ». La négociation apparaît désormais comme un trait culturel. Dans une société où l'individualisme est constamment valorisé, elle garantit la perpétuation d'une action collective. Cette transformation structurelle affecte la sphère politique. Les travaux d'Arend Lijphardt et de Robert Dahl marquent une reconsidération profonde du système démocratique. A partir de nombreuses études empiriques, Lijphardt est parvenu à dégager un schéma conceptuel (conceptual map) d'une grande portée reposant sur la distinction entre démocratie consensuelle et démocratie majoritaire ${ }^{5}$.

Ces apports théoriques permettent de mieux situer l'objet «Wikipédia ». En tant que dispositif d'écriture collaboratif, l'encyclopédie est un espace de négociation. Auray et al. (2009 : 46) y discernent un « lieu où se "marchandent" dans des négociations âpres les places respectives à attribuer aux points de vue concurrents. „ Ce marchandage constant entre différents points de vue s'effectue sur une vaste échelle. Les grandes communautés wikipédiennes comprennent plusieurs milliers de contributeurs actifs qui améliorent plus d'un million d'articles. En raison de leur ampleur, elles ne peuvent être abordées comme une somme de négociations micro-sociologiques : chaque communauté apparaît comme l'émanation d'une grande négociation structurelle et instituée.

Wikipédia peut être comprise comme une démocratie au sens où les entendent les études sur la négociation. Il existe une similarité profonde entre la dichotomie fondamentale de Lijphardt (consensuelle vs. majoritaire) et l'opposition wikipédienne entre le politique et le non-politique. La régulation de la communauté encyclopédique repose sur la possibilité donnée à chacun d'intervenir (l'édition des articles) et sur la pratique d'une rationalité communicationnelle (la discussion consensuelle). La démocratie consensuelle s'appuie un mécanisme proche : la pratique du pouvoir partagé (power sharing) garantit l'expression continue des principaux intérêts de la société civile ; elle implique une négociation constante qui fonde une rationalité démocratique supérieure à la simple rationalité du choix majoritaire (Lijphart, $1999: 2$ ).

\footnotetext{
${ }^{5}$ Pour une première expression de cette dichotomie fondamentale, cf. l'article séminal de Lijphart, 1969
} 
Serait-il possible de développer une théorie Lijphartienne de Wikipédia ? Si Wikipédia n'est pas une démocratie ou, plus exactement, une démocratie majoritaire au sens où l'entend Lijphart, comment se régule-t-elle au jour le jour ? Quels outils et quelles modalités décisionnelles ont été mis en place pour résoudre les problématiques propres à n'importe quelle société de grande taille (en moyenne plusieurs contributeurs réguliers) ?

Wikipédia ne s'intègre pas aisément dans l'appareil conceptuel de Lijphart : les typologies précises exposées dans Patterns of Democracy s'avèrent inopérantes. On ne peut mobiliser Lijphart qu'à un niveau d'appréhension assez large. Des apports complémentaires sont nécessaires pour resserrer la focale.

Les sciences de l'information et de la communication proposent un cadre méthodologique approprié à l'analyse de la gouvernance wikipédienne. Elles incitent à se focaliser sur la matérialité du support par-delà la multiplicité de ses représentations. II s'agit de relever en quoi ces dispositifs sous-jacent induisent certaines pratiques, certaines modalités de réception et certaines préférences sociales (Davallon, 2004 : 36). Or, l'encyclopédie se présente comme un projet socio-technique, où interactions sociales et infrastructures logicielles se conditionnent mutuellement (Jullien, 2012 : 3) : la structure technique est amendée en fonction de besoins communautaires préexistants ; elle affecte le comportement des utilisateurs en suscitant des réflexes éditoriaux durables. Nous sommes ainsi amené à considérer les débats wikipédiens dans la pluralité de leurs inscriptions éditoriales : à la fois émanation d'un état social (les relations propres à une communauté active de plusieurs milliers d'utilisateur), d'une construction technique (l'architecture logicielle du site) et d'une figuration sémiotique (l'articulation entre les différentes formes signifiantes).

Le recours à une méthodologie communicationnelle favorise un corpus restreint. ॥ comprend les principaux centres de décision de quatre grandes communauté wikipédiennes (anglophone, francophone, germanophone et italophone) : jusqu'en 2003, la liste de diffusion Wikipedia-I, puis les pages spécifiquement dédiées à l'évolution de la gouvernance interne (telles que « Wikipédia:Prise de décision » sur la Wikipédia francophone). Un outil propre au logiciel wiki, l'historique, permet de remonter dans le temps et de consulter la quasi-totalité des archives wikipédiennes. 


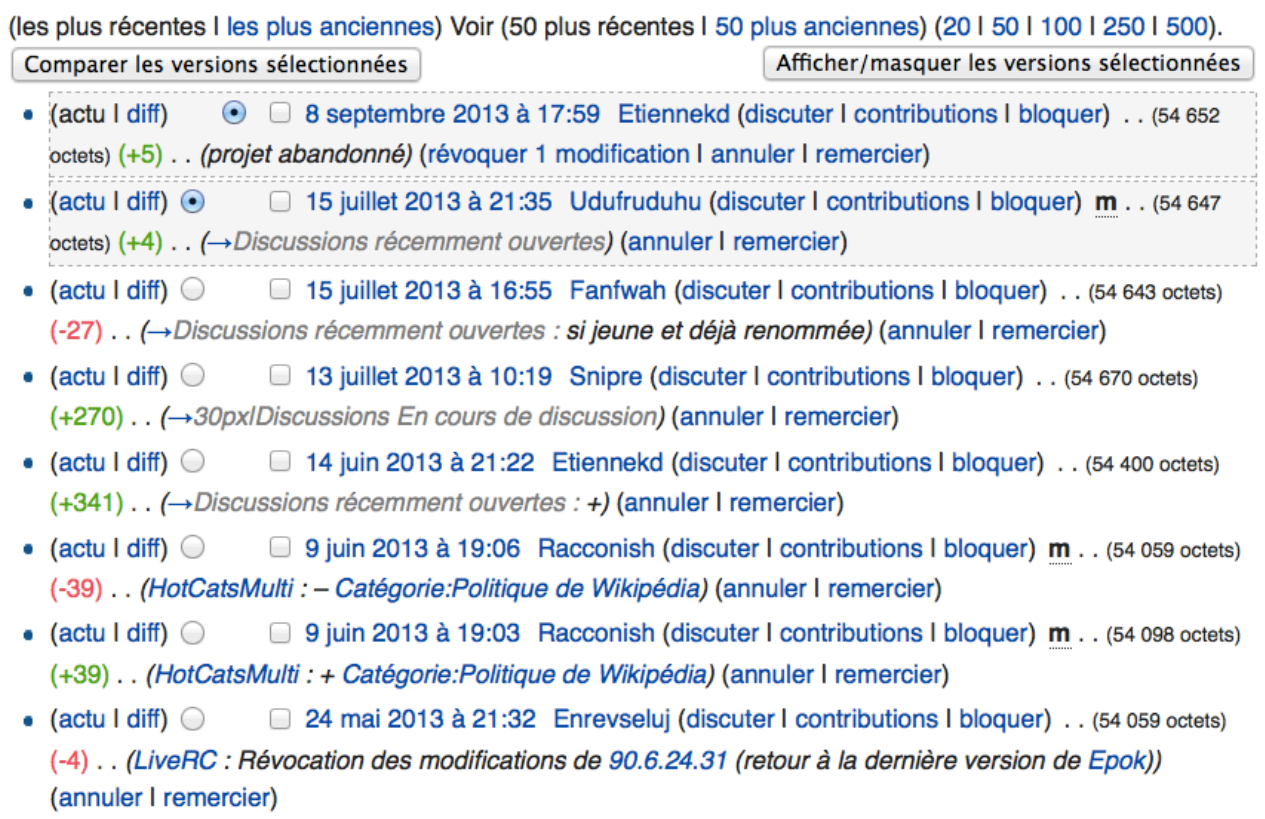

\section{Un exemple d’historique : celui de la page « Wikipédia:Prise de décision »}

Notre réflexion suit une ligne de développement diachronique. L'on s'intéressera aux débats originels sur l'identité de Wikipédia, et plus particulièrement à la tentative de synthèse développée par son co-fondateur, Jimmy Wales (1). Cette synthèse walesienne ne s'impose pas complètement. Dès 2002, les communautés non-anglophones se rallient à un modèle mixte, associant des pratiques de démocratie majoritaire à l'idéal d'une démocratie consensuelle (2).

\section{Un wiki encyclopédique? Pratique et idéologie d'une technique.}

Le wiki a d'abord été une invention conceptuelle. En 1990, Christine M. Neuwirth en donne une description par anticipation. Elle prévoit un système collaboratif (co-authoring) fonctionnant par paliers successifs : chaque participant ajouterait successivement sa contribution sur un texte donné (p. 183). Cinq ans plus tard, le WikiWikiWeb de Ward Cunningham actualise cette prévision ${ }^{6}$.

Cunningham conçoit moins le wiki comme un lieu pour la réflexion que comme un lieu pour l'action?. Toute discussion est d'abord un acte de discussion (speech act) : elle doit être préalablement publiée (Ortega, 2009 : 24). Chaque publication est consignée dans I'historique et reste constamment disponible. II n'y a jamais de résultat final qui correspondrait à l'aboutissement d'une réflexion : la conversation entre utilisateurs est un

\footnotetext{
6 «Wiki History » sur c2.com. Consulté le 14 janvier 2013 à l'adresse : http://c2.com/cgi/wiki?WikiHistory

7 Courrier de Ward Cunningham du 5 novembre 2012. Consulté le 14 janvier 2013 à l'adresse : http:// lists.wikimedia.org/pipermail/wiki-research-//2012-November/002572.html
} 
processus éternellement recommencé et éternellement réactivable (Reagle, 2010 : « Foreword »). Le wiki est en ce sens un lieu emblématique de "l'âge de la négociation »: il encourage une continuelle confrontation des opinions communes de ses utilisateurs.

Wikipédia se différencie dès sa création de la majorité des wikis. Le nouveau projet se définit comme une encyclopédie participative, qui vient compléter une autre encyclopédie en ligne, faisant classiquement appel aux contributions des experts : Nupedia (Ortega, 2009 : 9). La coalescence de deux entités sociales et techniques distinctes va rapidement susciter des frictions. A la fin du $\mathrm{XX}$ e siècle, le fonctionnement éditorial d'une encyclopédie repose sur une hiérarchie stricte. Les éditeurs déterminent le contenu et font appel à des auteurs reconnus pour leur expertise (ou à des employés à temps plein pour rédiger les articles de second rang) (Greenstein, 2009 : 9). Inversement, le wiki constitue une communauté de pratiques reposant sur une structure sociale ouverte (tout-le-monde peut la rejoindre) et égalitaire (tous les auteurs ont les même droits en ce qui concerne la modification de pages) (Jullien, $2012: 3$ ).

Quelques jours après la création de Wikipédia, un des premiers contributeurs soulève le problème suivant : que faire des discussions concernant les articles ? faut-il les déplacer dans une page à part ? Un autre contributeur pense que ce n'est pas nécessaire : sur un wiki tout se corrige de soi-même, les discussions trop étendues finiront par s'autoréguler ${ }^{8}$. Larry Sanger ne tient pas le même discours :

Je ne suis pas sûr que se référer à l'histoire du wiki soit très pertinent ici. Après tout, nous nous efforçons de créer une encyclopédie et, dès lors, nos articles ne peuvent pas être biaisés ${ }^{9}$.

Un éclaircissement devient nécessaire : Wikipédia ne peut continuer à perpétuer deux modalités socio-technique. Dans un courrier du 13 juin 2001, Jimmy Wales commence à fixer les grandes lignes organisationnelles du projet ${ }^{10}$. Deux notions centrales permettent d'esquisser un compromis entre wiki et encyclopédie : le consensus rationnel et le dictateur bienveillant (benevolent monarch).

Wales souligne d'abord que la communauté des auteurs ne peut se perpétuer que par le recours à un consensus informel capable d'induire des normes comportementales : 8 Courrier de Bryce Harrington du 27 janvier 2001. Consulté le 12 janvier 2013 à l'adresse http://
lists.wikimedia.org/pipermail/wikipedia-l/2001-January/000004.html

9 Courrier de Larry Sanger du 27 janvier 2001. Consulté le 12 janvier 2013 à l'adresse http:// lists.wikimedia.org/pipermail/wikipedia-//2001-January/000006.html

10 Courrier de Jimmy Wales du 13 juin 2001. Consulté le 12 janvier 2013 à l'adresse http:// lists.wikimedia.org/pipermail/wikipedia-//2001-June/000187.html 
L'une des propriétés les plus surprenantes de Wikipédia c'est que l'on parvient à d'excellents résultats sans recourir à des règles formelles ou à des restrictions. II y a des injonctions et des pressions sociales qui parviennent à garantir parfaitement le bon ordre des choses. ${ }^{11}$

Cette référence au consensus et au formatage social spontané est habile. Elle jette un pont entre les pratiques courantes de la communauté scientifique (l'échange rationnel comme outil procédural) et les modalités organisationnelles du logiciel wiki (égalité entre les participants, égalité d'accès etc.). Le consensus est une pratique courante dans les institutions informatiques et numériques (en particulier au sein du W3C ou de l'IETF) : s'y référer permet de se conformer à l'horizon d'attente des premiers wikipédiens (Reagle, 2010 : « Rough Consensus »).

Wales définit ensuite son rôle particulier : en tant que propriétaire et responsable du site il peut opérer des changements unilatéraux. Toutefois, cette capacité n'est pas absolue : il ne peut en faire qu'un usage pragmatique et bienveillant. Autrement, cela « tuerait l'essor du projet " et risquerait de décourager la participation communautaire ${ }^{12}$. Le dictateur bienveillant constitue ainsi une analogie affaiblie de l'éditeur encyclopédique : tout en gardant la main haute sur la gestion de la publication, il doit tenir compte des réactions communautaires.

Les circonstances particulières de la naissance de Wikipédia expliquent le rejet délibéré de l'interprétation politique. Le projet ne se présente ni comme une démocratie formelle ni comme une démocratie 2.0 afin de pérenniser son statut d'encyclopédie. L'aspect social inhérent à toute communauté fédérée par un wiki est refoulé. Dans une version plus approfondie de sa synthèse, Jimmy Wales précise que les discussions sur la nature de Wikipédia ne peuvent figurer qu'en dehors de Wikipédia (de préférence sur la liste de diffusion Wikipedia-I) : "Les articles de Wikipédia ne portent que sur des thématiques externes et non internes à Wikipédia $»^{13}$.

\section{Divergence des projets et modèle mixte}

Wales formalise sa synthèse dans un contexte assez particulier. En octobre 2001, Wikipédia n'est qu'un projet confidentiel, animé par 145 contributeurs actifs dont 115 sur la

\footnotetext{
11 lbid

${ }^{12}$ Courrier de Jimmy Wales du 13 juin 2001. Consulté le 12 janvier 2013 à l'adresse http:// lists.wikimedia.org/pipermail/wikipedia-//2001-June/000187.html
}

${ }^{13}$ Page « Jimbo Wales/Statement of Principles » sur nostalgia.wikipedia.org, version du 27 octobre 2001 (http://ppfr.it/e2o) 
seule Wikipédia anglophone ${ }^{14}$. Une communauté aussi restreinte n'a pas besoin d'une organisation précise. A vrai dire, il est même préférable de garder un certain flou sur la visée exacte du site afin d'encourager la participation : la préservation de la magie wiki (wiki magic) demeure une priorité.

Aujourd'hui, la synthèse walesienne reste appliquée au niveau social élémentaire, dans le cadre du travail de rédaction d'un ou de plusieurs articles - soit une situation qui n'a pas fondamentalement évolué depuis la naissance de Wikipédia. Le petit nombre de contributeurs concernés autorise la perpétuation d'une négociation souple et informelle (Cardon et Levrel, 2009 : 57). La collaboration encyclopédique locale met en application les grands principes généraux dégagés par Erhard Friedberg (1998). Elle dépend de règles déjà décidées à l'échelon communautaire et intégrées par les participants. Elle donne ensuite lieu à des interactions structurées par ce « jeu » déjà normé.

$\mathrm{Au}$ niveau de la gouvernance communautaire et de la concrétisation de normes collectives, la situation évolue rapidement dans une direction différente. Dès 2002, la synthèse walesienne perd du terrain. Les versions francophones et germanophones de l'encyclopédie prennent de l'importance. Elles divergent rapidement du modèle anglophone et se dotent de leurs propres normes participatives.

Le statut de dictateur bienveillant souffre de cette divergence. Wales ne peut pas perpétuer son rôle éditorial particulier dans des environnements linguistiques étrangers. Les communautés non-anglophones sont laissées à elles-même. En l'absence de toute autorité suprême, elles mettent en place leurs propres outils de régulation. L'évolution de la liste mail Wikipédia-I témoigne de cette délégation. Après 2002, elle commence à stagner :

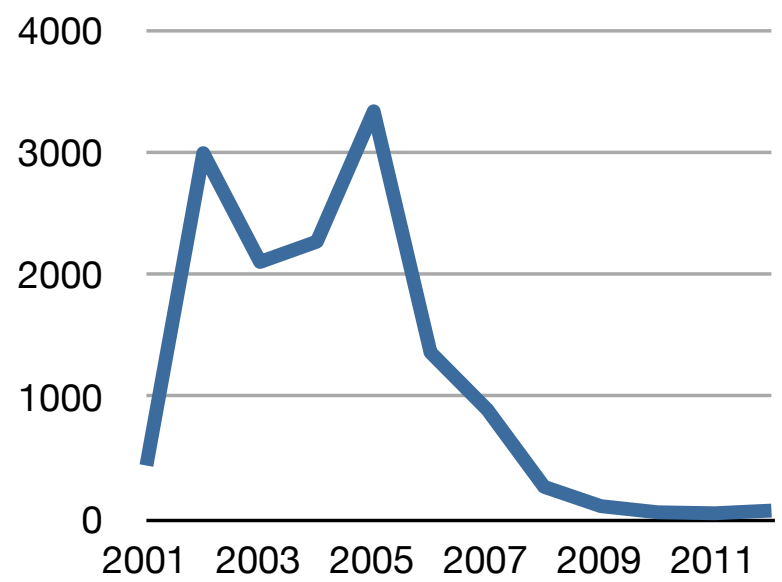

Taille de la liste Wikipedia-I (en KB) par année

14 Statistiques accessibles sur stats.wikimedia.org (http://ppfr.it/e2r \& $\underline{\text { http://ppfr.it/e2s) }}$ 
La Wikipédia francophone prend rapidement son destin en main. Le 7 octobre 2002, Florence Dévouard (alias Anthère) crée la page « Prise de décision ». Avec l'élargissement de la communauté, il « devient plus difficile de suivre toutes les pages ${ }^{15}$. Afin de gagner du temps, il importe de centraliser toutes les initiatives. Le choix final dépend d'un vote consensuel et non d'un simple consensus. L'une des premières prises de décision portait sur le choix d'un logo. Les avis se succèdent comme autant de bulletins électoraux mis en perspective :

\footnotetext{
7 pour anthere

6 pour Dave

6 pour Francis

6 pour meszigues

8 pour Rinaldum
}

Salut Rinaldum,

Ma préférée est la 7, mais la 6 sera ok (mais, elle apparait de qualité moins bonne, en bas, c est "tremblé", tu peux améliorer ca ?)

Par contre, il y a quelque chose qui me semble important

\section{Page Prise de décision, version du 2 novembre 2002 (http://ppfr.it/e1r)}

Pour autant il ne s'agit pas d'un vote classique. On ne cherche pas seulement à identifier les positions de chacun mais à poser les conditions d'un débat rationnel :

Le système de vote étant consensuel, vous êtes invités à faire de nouvelles propositions pour faire avancer les choses et à modifier votre vote si de nouvelles propositions vous conviennent. ${ }^{16}$

Dans l'illustration ci-dessus, le vote est suivi de plusieurs remarques discursives, qui permettent d'esquisser une solution consensuelle. Tout en rappelant que le logo $n^{\circ} 7$ a sa préférence, un contributeur admet que le logo $n^{\circ} 6$ constitue un choix acceptable. II en profite pour énoncer quelques recommandations (améliorer la résolution de l'image).

La prise de décision francophone esquisse ainsi un modèle mixte : il ne s'agit pas d'un consensus à l'état pur tel que préconisé par Wales ; il ne s'agit pas non plus d'un simple constat du choix majoritaire. Cette articulation entre deux formes de gouvernance n'aurait pas surpris Lijphart. En introduction de Patterns of democracy (1999: 7-8), il remarque qu'il n'existe pas de démocratie majoritaire ou consensuelle parfaite : chaque État intègre

\footnotetext{
15 Page «Wikipédia:Prise de décision/Logo du Wikipédia francophone » de fr.wikipedia.org, version du 5 novembre 2002 (http://ppfr.it/e2c)

${ }^{16}$ Page « Wikipédia:Prise de décision » de fr.wikipedia.org, version du 7 octobre 2002 (http://ppfr.it/e2d)
} 
des traits de l'une et de l'autre selon des proportions variables. On ne peut leur affecter qu'un positionnement relatif, allant du plus consensuel (la Suisse, les Pays-Bas...) au plus majoritaire (le Royaume-Uni, la Nouvelle-Zélande...).

Aujourd'hui, les communautés encyclopédiques de grande ampleur (plus de 1000 contributeurs réguliers) recourent à diverses déclinaisons du modèle mixte. La synthèse Walesienne a partout été amendée, sous la pression d'un certain pragmatisme. Elle pose en effet deux problèmes fondamentaux : dans un espace encyclopédique comprenant des millions de pages les décisions manquent de visibilité ; à impliquer trop de monde, les discussions finissent par traîner en longueur (Reagle, 2010 : « Polling and voting »).

Significativement, les votes communautaires sont présentés comme des raccourcis. Sur la Wikipédia italophone, le Sondaggio se veut un « mécanisme simple et rapide pour résoudre facilement les problèmes actuels. " ${ }^{17}$ Les wikipédiens germanophones se sont résolus à instituer le Meinungsbilder pour « clarifier les questions sur lesquelles aucun consensus n'a pu être atteint. " ${ }^{18}$ En apparence la Wikipédia anglophone reste fidèle au consensus pur. Néanmoins, depuis plusieurs années, les Appels à commentaires (Request for comments) tendent à incorporer des éléments de vote (avis Keep ou Oppose en gras).

Here's what I propose:

If the victim has received coverage in an exclusive manner by at least two different, independent, and secondary reliable sources, regardless of the length of the content of said coverage, the victim is considered notable enough to merit an article on Wikipedia.

That would be so specific that it won't be subject to interpretation and discussions like this won't happen ever again.

What do you guys think?

-Ahnoneemoos (talk) 14:00, 11 January 2013 (UTC)

- Oppose. A guideline being subject to interpretation is not necessarily a bad thing. Phrases like "persistent coverage" can quite legitimately be discussed by editors in relation to individual cases (may I also draw your attention to the uncertain meaning of "in an exclusive manner", BTW). The basic purpose of the guidance is to set a bar so that not every victim of a crime that has appeared in the news becomes the subject of a WP article. Your proposal may as well be to just remove the guidance, because it basically boils down to GNG. That's not to say there's no room for improvement in the current wording. Formerip (talk) 14:12, 11 January 2013 (UTC)

\section{Request for Comments de la page Wikipedia talk:Notability (people) Version du 12 janvier 2013 (http://ppfr.it/e1q)}

\footnotetext{
17 Page « Wikipedia:Sondaggio » de it.wikipedia.org, version du 28 juillet 2004 (http://ppfr.it/e21)

18 Page « Wikipedia:Meinungsbilder » de de.wikipedia.org, version du 13 janvier 2013 (http://ppfr.it/e22)
} 
L'évolution de la gouvernance wikipédienne pourrait se résumer à une dialectique constante entre légitimité et efficacité. Le cas francophone est exemplaire de cette adaptabilité. Chaque prise de décision définit ses propres modalités d'acceptation, lesquelles donnent lieu à une longue discussion préalable (elle peut parfois durer jusqu'à neuf mois). Généralement, le choix se fait à la majorité qualifiée $(55,60 \%$ et $75 \%$ sont les valeurs les plus courantes). Lorsque plusieurs propositions sont en lice, on recourt fréquemment à la méthode Condorcet, qui discrimine la proposition qui convient à le plus de monde plutôt que celle qui réunit le support le plus large :

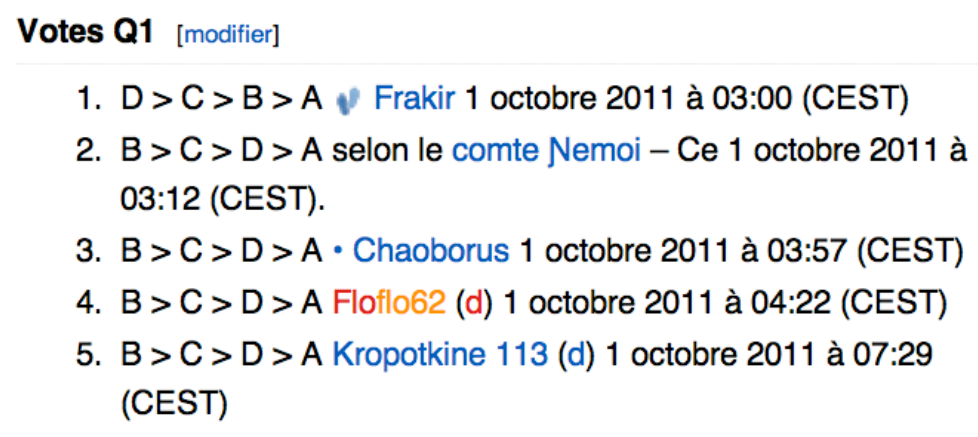

\section{Extrait de la Prise de décision sur la contestation du statut d'administrateur Version du 16 octobre 2011 (http://ppfr.it/e1s)}

Dans la mesure où les votes sont plus des outils que des sources de légitimité, leur statut change au gré des besoins effectifs de la communauté. Depuis 2007, les prises de décision sont de moins en moins utilisées sur la Wikipédia francophone. La validation communautaire passe désormais de plus en plus par une procédure moins formelle : le sondage ${ }^{19}$. En 2012, l'on dénombre 32 sondages contre seulement deux prises de décision adoptées. La mutation n'est pas seulement quantitative. Les thématiques du style enquête d'opinion (par exemple Vouvoyer ou tutoyer sur Wikipédia ?) disparaissent au profit de questions plus élaborées et plus conséquentes : réforme des critères d'admissibilité des articles, mise en place d'une campagne d'information à destination des lecteurs...

19 Page « Wikipédia:Sondage » de fr.wikipedia.org dans sa version du 2 avril 2004 (http://ppfr.it/e3b) 


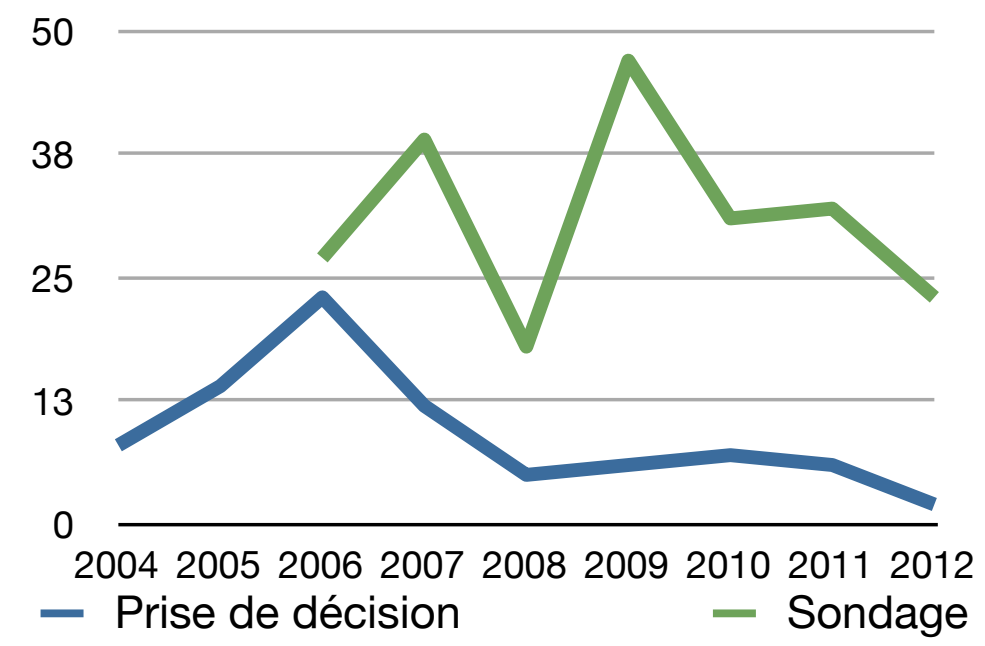

\section{Évolution comparée des systèmes de vote sur fr.wikipedia.org}

Qu'advient-il des décisions une fois adoptées ? Dès lors que leur clarté le permet, elles sont appliquées scrupuleusement. Cardon et Levrel (2009: 57 et 87) mettent en évidence un mécanisme de vigilance participative qui facilite grandement la diffusion des normes communément définies à l'ensemble de l'espace encyclopédique. Les relations de contributeurs à contributeurs reposent sur une confiance et/ou une surveillance mutuelle : chaque wikipédien est potentiellement coupable, juge, témoin et policier (Geiger, 2007 : 31).

\section{Du bricolage en politique...}

Si l'on devait répondre en une phrase à notre questionnement initial, ce serait celle-ci : les communautés wikipédiennes ont fait le choix du flou, soit d'une indétermination volontaire entre les différents modèles d'organisation possibles. Le refus apparemment ferme du politique et de la démocratie a une valeur heuristique. II met en sourdine les débats idéologiques sur le statut de l'encyclopédie. L'appropriation par les communautés des procédures décisionnelles de l'État moderne se fait sur une base pragmatique : le vote est un outil et non un fondement symbolique ; si l'outil n'est pas suffisamment probant, il est toujours possible de l'améliorer en injectant des éléments de négociation et de rationalité communicationnelle.

II ne faut pas prendre ici le flou en un sens péjoratif ${ }^{20}$. Selon Boltanski et Thevenot (1991 : 50), les associations humaines ne peuvent se maintenir qu'en maintenant une certaine incertitude sur leur raison d'être. Des représentations et normes collectives trop

20 On retrouve un peu cet a priori dans Geiger $(2007: 83)$ 
unilatérales risquent de décourager des adhésions potentielles : le recrutement de nouveaux membres repose nécessairement sur une part de méprise. Chaque projet encyclopédique se trouve constamment en état d'équilibre fluctuant : un contributeur francophone décrit un « projet qui tourne très bien alors qu'il prouve tous les jours qu'il ne devrait logiquement pas fonctionner $»^{21}$.

À partir de cette conclusion générale, il est possible d'énoncer deux principales contributions de notre analyse aux études sur la négociation.

Sur un plan expérimental, les projets encyclopédiques constituent de bons candidats à un élargissement de l'approche lijphardtienne. Les principales critiques de Patterns of Democracy portent sur la faiblesse de l'échantillon : pour Gaughof (2010) les 36 démocraties sélectionnées ne constitueraient pas un panel suffisant pour aller au-delà d'une simple herméneutique. L'échantillon n'est pas indéfiniment extensible. II n'existe actuellement que 200 expériences étatiques (peut-être le double si l'on tient compte des entités fédérées). Cela reste sans doute insuffisant pour tester et approfondir le schéma conceptuel de Lijphart. Or les communautés en ligne de grande taille constituent des objets analogues aux systèmes politiques formels, capable qui plus est de faire émerger des enjeux spécifiques. L'unicité apparente de Wikipédia dissimule une dizaine voire une vingtaine de communautés linguistiques autonomes, qui ont chacune institué leur propre cadre procédural.

Sur un plan théorique, l'analyse confirme in fine certaines orientations récentes des études sur la négociation en démocratie. Le modèle de la démocratie consensuelle tend à montrer ses limites. Giraud (2009 : 220) souligne qu'il n'est pas davantage épargné par la crise de la représentation politique que la démocratie majoritaire. Par contraste, de nouveaux modèles composites s'affirment. La Suisse réalise une heureuse synthèse de la démocratie directe et de la démocratie délibérative. Le déclin des intermédiaires traditionnels est compensé par l'émergence d'un débat public continu, impliquant toutes les couches de la société. L'utilisation régulière du référendum permet de s'assurer que la négociation ne reste pas entre les mains d'une élite politique ou sociale (Giraud, 2009 : 223).

La gouvernance wikipédienne illustre, dans une certaine mesure, ce modèle composite, qualifié par Giraud de « démocratie délibérative populaire ». La discussion rationnelle ouverte (au sens où elle implique potentiellement tous les participants) et l'assentiment communautaire constituent les deux principales sources de légitimité des décisions

21 Extrait du sous-titre du blog Le Choix du chaos. Consulté le 14 janvier 2013 (http:// choixduchaos.blogspot.fr/) 
collectives. Même si des personnalités charismatiques peuvent peser sur son orientation, le débat public n'est jamais capté par une petite minorité spécialisée.

Signe de cette convergence, la coupure entre Wikipédia et le monde politique traditionnel n'est plus si nette. Plusieurs mouvances émergentes citent l'organisation wikipédienne comme un exemple à suivre. Publié en 2012, l'ouvrage collectif The Wealth of the Commons vise à théoriser le mouvement des biens communs. II inclut de nombreuses références à Wikipédia, à la fois en tant que mode de production éditoriale de pair-à-pair et comme système de préservation auto-géré d'une ressource commune (Le Crosnier, 2012).

Paradoxalement, une communauté qui prétendait se tenir à l'écart des activités politique contribue aujourd'hui à enrichir la réflexion politique.

\section{Références}

AURAY Auray et al. (2009), "La négociation des points de vue : une cartographie sociale des conflits et des querelles dans le Wikipédia francophone ", Réseaux $n^{\circ} 154$, 2009/2, pp. 15-50

BLACK Laura et al. (2008), "Wikipedia is Not a Democracy": Deliberation and PolicyMaking in an Online Community », conférence présentée lors du meeting annuel de I'International Communication Association, Montréal / Consulté le 25 octobre 2013 à l'adresse http://www.cs.cornell.edu/ danco/research/papers/democracy-black-ica2008.pdf

BOLTANSKI Luc \& Laurent THÉVENOT (1991), De la justification — Les économies de la grandeur, Paris, Gallimard.

BUTLER Brian et al. (2008), « Don't Look Now, But We've Created a Bureaucracy: The Nature and Roles of Policies and Rules in Wikipedia ", conférence présentée lors de l'édition 2008 du Computer Human Interaction, Florence.

CARDON Dominique \& JULIEN LeVREL (2009), « La vigilance participative. Une interprétation de la gouvernance de Wikipédia », Réseaux $n^{\circ} 154$, 2009/2, pp. 51-89.

DAVALLON Jean (2004), « Objet concret, objet scientifique, objet de recherche » Hermès, $n^{\circ} 38,2004$, p. 30-38.

FRIEDBERG Ehrard (1998), Le Pouvoir et la règle : dynamiques de l'action organisée, Paris, Seuil. 
GEIGER R. Stuart (2007), Democracy in Wikipedia, Senior Thesis soutenue à l'University of Texas, Austin, 2007.

GAUGHOF Steffen (2010), « Democratic Inclusiveness: A Reinterpration of Lijphart's Patterns of Democracy », British Journal of Science, vol. 40, n³, 2010, pp. 679-692.

Greenstein Shane et Michelle DevereuX (2009), « The Crisis at Encyclopædia Britannica », Étude de cas pour la Kellogg School of Management, 2009.

JULLIEN Nicolas (2012), What we know about Wikipedia. A review of the literature analyzing the project(s), publié le 7 mai 2012, dernièrement modifié le 2 octobre 2012. Consulté à l'adresse http://papers.ssrn.com/sol3/papers.cfm?abstract id=2053597

LE Crosnier Hervé (2012), "Subtle But Effective: Modern Forms of Enclosures », dans Bollier Daniel et Silke Helfrich (ed.) The Wealth of the Commons, Levellers Press.

Consulté en ligne le 16 avril 2014 à l'adresse http://wealthofthecommons.org/essay/subtleeffective-modern-forms-enclosures

LIJPHART Arendt (1999), “Consociational Democracy », World Politics, vol. 21, 1969/2, pp. 207-225.

LIJPHART Arendt (1999), Patterns of Democracy, Yale University Press.

NEUWIRTH Christine et al. (1990), "Issues in the desing of computer support for coauthoring and commenting " dans CSW'90: Proceedings of the 1990 ACM conference on Computer-supported cooperative work, New York, ACM Press, pp. 183-195.

ORTEGA Felipe (2009), Wikipedia: A quantitative analysis, thèse de doctorat en télécommunication, soutenue à l'Université Rey Juan Carlos de Madrid en mars 2009.

REAGLE Joseph (2010), Good Faith Collaboration, Cambridge, MIT Press. Mis en ligne sur http://reagle.org/joseph/2010/gfcl, consulté le 14 janvier 2013

TOUZARD Hubert (2009), « Acquis et perspectives de la recherche sur la négociation ", Négociations, $\mathrm{n}^{\circ} 12$, pp. 60-6.

VEDEL Thierry (2006), «La révolution ne sera plus télévisée. Internet, information et démocratie ", Pouvoirs, n¹19, 2006, pp. 41-54.

ZARTMAN Ira William (1976), The 50\% Solution: How to Bargain Successfully with Hijackers, Strikers, Bosses, Oil Magnates, Arabs, Russians, and Other Worthy Opponents in This Modern World, Anchor Press. 